\title{
Evaluation of endothelial function and cardiovascular risk in non-obese patients with slight degree of obstructive sleep apnea syndrome
}

\author{
Fiorella Devito1, Annapaola Zito1, Silvano Dragonieri2, Pierluigi Carratù'2, \\ Vitaliano N. Quaranta2, Francesco Vitale3, Nicola Quaranta3, Francesco Panza4, \\ Giancarlo Logroscino4, Matteo Cameli ${ }^{5}$, Onofrio Resta ${ }^{2}$, Marco M. Ciccone ${ }^{1}$ \\ 1Section of Cardiovascular Diseases, Department of Emergency and Organ Transplantation, University \\ of Bari "Aldo Moro", Italy \\ 2Section of Pulmonary Disease, University of Bari "Aldo Moro", Italy \\ 3Otolaryngology Unit, Department of Basic Medical Science, Neuroscience and Sensory Organs, \\ University of Bari "Aldo Moro", Italy \\ 4Neurodegenerative Disease Unit, Department of Basic Medicine, Neuroscience, and Sense Organs, \\ University of Bari “Aldo Moro", Italy \\ 5Department of Public Health and Clinical Medicine, Heart Centre, Umeå University, Umeå, Sweden
}

\begin{abstract}
Recently, it has been clearly described an independent relationship between obstructive sleep apnea syndrome (OSAS) and cardiovascular risk, with underlying mechanisms also including endothelial dysfunction. We enrolled 32 consecutive non-obese patients (mean age of $39.5 \pm 11.5$ years), of which 16 with mild OSAS and 16 snoring without OSAS. Mild OSAS is defined by an AHI index between 5 and 15 . We have investigated if whether there was a relationship between mild OSAS, endothelial function and carotid intima-media thickness (C-IMT).
\end{abstract}

Corresponding author: Pierluigi Carratù, Institute of Respiratory Diseases, University of Bari "Aldo Moro", Policlinico, Piazza Giulio Cesare 11, 70124 Bari, Italy. E-mail: pierluigi.carratu@uniba.it

Key words: Obstructive sleep apnea syndrome; carotid intima-media thickness; endothelial function; cardiovascular risk; flow mediated dilation; CPAP.

Contributions: FD, VQ, manuscript drafting; AZ, FV, MC, literature searches managing; SD, FP, GL, figures design, literature searches managing, contribution to draft correction; $\mathrm{PC}$, patients enrolling; NQ, OR, MMC, figures provision, work supervision. All authors read and approved the final manuscript.

Conflict of interest: The authors declare no conflict of interest.

Received for publication: 24 March 2017

Accepted for publication: 5 November 2017

(C) Copyright F. Devito et al., 2017

Tipografia PI-ME Editrice, Italy

Monaldi Archives for Chest Disease 2017; 87:822

doi: $10.4081 /$ monaldi.2017.822

This article is distributed under the terms of the Creative Commons Attribution Noncommercial License (by-nc 4.0) which permits any noncommercial use, distribution, and reproduction in any medium, provided the original author(s) and source are credited.
The population was divided into two groups: Group 1 (16 simple snorer patients with an average age of $39.4 \pm 12.1$ years) and Group 2 (16 subjects with mild OSAS with an average age of 39.6 \pm 11.2 years). Each group underwent cardiovascular investigation including measurement of flow-mediated dilation (FMD) of the brachial artery and C-IMT. Both groups comprised non-obese subjects. Patients with mild OSAS had serum total cholesterol values statistically significantly higher than simple snores patients $(178.6 \pm 24.9$ vs $159.2 \pm 25.3$; $\mathrm{p}=0.038)$. OSAS patients had also a trend towards higher values of maximum C-IMT compared to simple snorer patients $(0.70 \pm 0.15$ vs $0.65 \pm 0.16)$, although below the level of significance. Between the two groups, no difference was found for FMD values. The present results on mild OSAS strengthen the importance of a diagnosis of OSAS as soon as possible, in order to encourage all primary prevention interventions to correct risk factors responsible for disease progression and the occurrence of cardiovascular diseases, not excluding the use of therapies of noninvasive ventilation even in the early stages of the disease.

\section{Introduction}

The study of endothelial function is an interesting research tool to evaluate subclinical atherosclerosis and the assesstment of endothelial function could improve the evaluation of cardiovascular risk; in other words, endothelial dysfunction is associated to an increased cardiovascular risk. The endothelium is considered as a real organ with autocrine and paracrine capabilities, designed to modulate vascular tone and structure. In fact, in physiological conditions, the synergistic action of endothelial factors allows the maintenance of normal vascular tone and blood fluidity while limiting inflammatory damage of the vessel and the proliferation of smooth muscle cells. In pathological conditions, the endothelium changes its characteristics by promoting phenomena of inflammation and thrombosis, losing control of active vascular permeability and ultimately promoting vasoconstriction [1].

Endothelial dysfunction can be caused by many factors, including the obstructive sleep apnea syndrome (OSAS). OSAS is a very common condition, recognized as a major public health burden [2]. OSAS is characterized by apnoea or hypopnoea that lasts for a minimum of 10 seconds. Apnoea is the complete cessation of airflow for at least $10 \mathrm{sec}$ onds. Hypopnoea is characterized by the drop of $30 \%$ of airflow for 10 
seconds if it is associated with $\geq 4 \%$ oxygen desaturation [3-5]. Since the muscle tone of the body normally relaxes during sleep, and since, at the level of the throat, the human airway walls are constituted by soft tissue, which might collapse, it is easy to understand why breathing can be obstructed during sleep, especially in obese subjects [4].

The main symptoms of OSAS are nocturnal respiratory pauses interrupted by loud intermittent snoring and excessive daytime sleepiness [3]. Other symptoms are: headache upon awakening, reduced memory and concentration capacity, dry mouth on awakening, restless sleep and several micro-arousals, enuresis, sexual impotence, gastroesophageal reflux [6], changes in mood, depression and anxiety [7]. Diagnosis of OSAS consists in minimum 5 events/hour of sleep time in association with clinical symptoms, or at least 15 events/hour of sleep time with or without clinical symptoms [8]. Treatment of OSA significantly improves early signs of atherosclerosis, supporting the concept that OSA is an independent risk factor for atherosclerosis [9].

Important studies have shown an independent relationship between OSAS and cardiovascular risk, with different mechanisms implicated: hypoxia, hypercapnia, micro-arousals, sympathetic hyperactivity, oxidative stress, systemic inflammation, or hypercoagulability. All these mechanisms lead to inactivation of the nitric oxide by endothelial increased production of free radicals of oxygen in the arterial wall [10]. Oxidative stress is considered to be the most important mechanism of endothelial dysfunction. Apnea is also responsible for the increase in systemic blood pressure, often refractory to treatment, and for thickening of carotid intima-media thickness (C-IMT). There is also the evidence that OSAS is correlated with insulin resistance and impaired glucose tolerance, which are factors that can support the alterations of the vessel walls. Increasing body of scientific evidence suggested a correlation between severity of OSAS and endothelial dysfunction, however this correlation was studied only in obese patients. [11-15]. The aim of the present study was to evaluate the existence of an association between a mild OSAS state in nonobese patients (BMI $<30$ ), the presence of endothelial dysfunction and vascular wall alterations. We focused on a very early stage of the OSAS that usually results unrecognized as compared to the more acclaimed stages but which is the subclinical precursor.

\section{Materials and Methods}

The present study was performed between September 2014 and September 2015 in the Pneumology Unit with the collaboration of the Angiology Section of the Cardiology Unit and the Otolaryngology Unit of Ospedale Policlinico, University "Aldo Moro" of Bari, Italy. Thirty-two OSA suspected patients were enrolled. Criteria for inclusion in the study were: BMI $<30$, diagnosis of mild OSAS [apnea-hypopnea index (AHI) <15], absence of smoke. Exclusion criteria from the study were represented by BMI $>30$, presence of asthma, chronic obstructive pulmonary disease or other comorbidities, the presence of $\mathrm{AHI}>15$, the presence of heart diseases (coronary heart disease, arrhythmia, heart failure), vascular brain diseases (stroke or a transient ischemic attack), peripheral obstructive artery disease (intermittent claudication, delayed or absent peripheral pulses), chronic liver disease, known kidney disease or any other chronic severe disease. For the present study, we enrolled two groups of patients: the first group consisted of 16 simple snorer patients (Group 1, control group), while a second group consisted of 16 patients who received the diagnosis of mild OSAS (Group 2) (Table 1). Each group was subjected to clinical and instrumental diagnosis in accordance with the guidelines of the American Academy of Sleep Medicine [16]; after patients underwent cardiovascular investigation including measurement of flow-mediated dilation (FMD) of the brachial artery and C-IMT.

\section{Clinical and laboratory examination and cardiorespiratory nocturnal monitoring}

Cases of type 2 diabetes mellitus, hypertension, and dyslipidemia (hypertriglyceridaemia and /or hypercholesterolaemia) were identified with a 2-phase procedure, utilizing clinical criteria described in detail elsewhere. Hypertriglyceridaemia has been defined by triglycerides more than $150 \mathrm{mg} / \mathrm{dL}$ and hypercholesterolaemia by low-density lipoprotein-cholesterol (LDL-C) $>70 \mathrm{mg} / \mathrm{dl}$ [17].

According to European Society of Cardiology, hypertension is defined as values of systolic blood pressure $\geq 140 \mathrm{mmHg}$ and/or values of diastolic blood pressure $\geq 90 \mathrm{mmHg}$ [18].

The diagnosis of diabetes was based, according to the World Health Organization criteria on glycated haemoglobin AlC $\geq 6.5 \%$ (48 mmol/mol) and fasting plasma glucose $\geq 7.0 \mathrm{mmol} / \mathrm{L}$ ( $\geq 126 \mathrm{mg} / \mathrm{dL}$ ) or 2-h post-load plasma glucose concentrations $\geq 11.1 \mathrm{mmol} / \mathrm{L}(\geq 200 \mathrm{mg} / \mathrm{dL})$ [19].

BMI was calculated as weight/height ${ }^{2}\left(\mathrm{~kg} / \mathrm{m}^{2}\right)$. Blood samples were obtained early in the morning after a 13 -hour overnight fast. Serum total cholesterol, triglycerides, high-density lipoprotein (HDL) cholesterol, and glycemia were measured by standard enzymatic-colorimetric methods. HDL cholesterol was measured in the supernatant after precipitation of apolipoprotein-B-containing particles with phosphotungstic acid and magnesium ions. LDL-C was calculated with the Friedewald formula [20]. The diagnosis of mild OSAS was made through polysomnography, the gold standard for the diagnosis. Cardiorespiratory nocturnal monitoring was performed in ambient air and spontaneous breathing using a portable 4-channels/8-tracks polysomnographer (Somnea Italy). In detail, oxyhaemoglobinic saturation, heart rate, body posture, oro-nasal air flow, snoring sounds, thoracic and abdominal movements were recorded. AHI, Oxyhaemoglobin Desaturation Index (ODI) and \% time of oxyhaemoglobin saturation spent below $90 \%$ (TST 90 ) were calculated. OSAS was defined by $\mathrm{AHI} \geq 5$ in presence of hypnologic symptoms or by $\mathrm{AHI} \geq 15$ without symptoms. The diagnosis of mild OSAS was made according to the guidelines of American Academy of Sleep Medicine [21-22] on the basis of: AHI between 5 and 14, oxygen saturation at least $86 \%$, and minimal disability morning. Drowsiness by modest degree can occur while watching television, or reading a book in a quiet room, or you are on a moving vehicle as a passenger. A drowsiness of this type may not occur every day. The AHI is the parameter currently used to assess the degree of severity of OSAS and is given by the number of apneas /hypopneas per hour of sleep calculated with a night recording system (polysomnography).

\section{FMD of the brachial artery}

All patients underwent to the measurement of FMD of the brachial artery under standardized conditions. This ultrasound method measures the ability of the brachial artery to dilate in response to increased blood flow and more specifically to the "shear stress". The primary mediator of FMD is NO produced by the endothelium. It is a non-invasive technique and allows repeated measurements. FMD was influenced by food, stress, temperature, drugs and sympathetic stimuli. This assessment was performed with the subjects fasting for at least 8-12 hours, early in the morning, in a quiet air conditioned room $\left(22-24^{\circ} \mathrm{C}\right)$. The subjects were asked not to play exercise or take exciting substances like chocolate or coffee/tea, which could impair endothelial function for at least 4-6 hours before the exam. A preliminary scan explored the anatomy and identified landmarks, paying attention to the presence of calcifications, arterial tortuosity or atherosclerotic plaques. The right brachial artery was evaluated in a long axis projection between 5 and $10 \mathrm{~cm}$ above the elbow. All patients 
were evaluated by the same operator to reduce the bias, by means of a high-resolution ultrasound device Philips Sonos 5500 with a 7.0 $\mathrm{MHz}$ or higher linear probe connected to an image analysis system, certified by the National Research Council of Italy (CNR) of Pisa (MVE II) [23], for calculating the brachial artery diameter in real time by analyzing B-mode ultrasound images, setting positivity to the test value at less than $5 \%$. The arm was positioned comfortably in order to get satisfying images of the brachial artery. The selected artery segment was above the antecubital fossa in the longitudinal plane. With the subject in supine position for at least $10 \mathrm{~min}$, a sphygmomanometer cuff was placed in the distal site to the artery, in cases of a humeral artery on the forearm. After 1 min of flow image baseline acquisition, the artery was occluded by inflating the cuff to a pressure of $200-220 \mathrm{mmHg}$ for $5 \mathrm{~min}$. When the cuff is deflated, it induces a short state of high flow (reactive hyperemia in the forearm microcirculation) through the brachial artery to adjust to the reduced downstream resistance caused by the ischemia-induced dilatation. The resulting increased "shear stress" provides the stimulus for dilatation of the humeral artery. Within 15 seconds from the end of ischemia, the flow rate was measured. Reactive hyperemia was calculated as the ratio of the change in diameter (maximal dilatation after deflation baseline) divided by the baseline value, which corresponds to the maximum FMD recovery value. FMD was analyzed as the percentage increase in brachial artery diameter after the application of a pressure stimulus [24].

\section{C-IMT ultrasonography}

All patients underwent also to two-dimensional echo-color Doppler of the carotid arteries. During the procedure, patients remained supine with neck extended and rotated contralateral to the probe of about $45^{\circ}$. C-IMT was defined as a low-level echogrey band that does notproject into the arterial lumen and was measured during end-diastole according to the Pignoli method: by focusing and freezing images on the distal wall of the common carotid artery on the lengthwise axis, the C-IMT was the distance between the leading borders of the first hyperechoic line and of the second hyperechoic line, separated by a hypoechoic space "doubletrack pattern" [25]. The measurements were performed for three times, bilaterally $1 \mathrm{~cm}$ proximally to the carotid bulb, and then C-IMT value was calculated as the arithmetical mean of each side. The IMT includes endothelial cells, connective tissue and smooth muscle cells and is the seat of lipid deposits when forming the atheromatous plaque. In normal adults, C-IMT values ranges from $0.25 \mathrm{~mm}$ to $1.5 \mathrm{~mm}$, but values $>0.9 \mathrm{~mm}$ are defined pathological.

\section{Statistical analysis}

Baseline demographic variables (age and gender), presence of cardiovascular risk factors (diabetes, hypertension, or dyslipidemia), and measurements of BMI, FMD and C-IMT were collected for each patient

Table 1. Demographic and clinical characteristics of patients with mild obstructive sleep apnea syndrome (OSAS) and simple snorers. Values are reported as mean \pm standard deviation for continuous variables and as numbers with a for categorical variables.

\begin{tabular}{|c|c|c|c|c|}
\hline & Simple snorers / Mild OSAS & N & Media or N (\%) & Significance \\
\hline Age & $\begin{array}{l}1 \\
2\end{array}$ & $\begin{array}{l}16 \\
16\end{array}$ & $\begin{array}{l}39.37 \pm 12.05 \\
39.56 \pm 11.23\end{array}$ & 0.964 \\
\hline Sex, males (\%) & $\begin{array}{l}1 \\
2\end{array}$ & $\begin{array}{l}16 \\
16\end{array}$ & $\begin{array}{l}8(50) \\
8(50)\end{array}$ & 0.638 \\
\hline Body mass index & $\begin{array}{l}1 \\
2\end{array}$ & $\begin{array}{l}16 \\
16\end{array}$ & $\begin{array}{l}22.75 \pm 3.19 \\
25.90 \pm 4.81\end{array}$ & 0.067 \\
\hline Total cholesterol (mg/dL) & $\begin{array}{l}1 \\
2\end{array}$ & $\begin{array}{l}16 \\
16 \\
\end{array}$ & $\begin{array}{l}159.21 \pm 25.32 \\
178.62 \pm 24.88\end{array}$ & 0.038 \\
\hline HDL cholesterol (mg/dL) & $\begin{array}{l}1 \\
2 \\
\end{array}$ & $\begin{array}{l}16 \\
16 \\
\end{array}$ & $\begin{array}{l}46.37 \pm 14.53 \\
50.62 \pm 12.82\end{array}$ & 0.387 \\
\hline LDL cholesterol (mg/dL) & 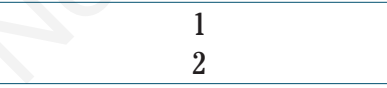 & $\begin{array}{l}16 \\
16\end{array}$ & $\begin{array}{c}88,37 \pm 17.13 \\
108.81 \pm 28.76\end{array}$ & 0.120 \\
\hline Triglycerides (mg/dL) & $\begin{array}{l}1 \\
2\end{array}$ & $\begin{array}{l}16 \\
16\end{array}$ & $\begin{array}{l}103.31 \pm 43.87 \\
101.62 \pm 21.09\end{array}$ & 0.891 \\
\hline Glycemia (mg/dL) & $\begin{array}{l}1 \\
2\end{array}$ & $\begin{array}{l}16 \\
16\end{array}$ & $\begin{array}{c}88.19 \pm 12.76 \\
83.00 \pm 8.19\end{array}$ & 0.181 \\
\hline T2DM (\%) & $\begin{array}{l}1 \\
2\end{array}$ & $\begin{array}{l}16 \\
16 \\
\end{array}$ & $\begin{array}{c}2(12.5) \\
1(6.3)\end{array}$ & 0.500 \\
\hline Hypertension (\%) & $\begin{array}{l}1 \\
2 \\
\end{array}$ & $\begin{array}{l}16 \\
16 \\
\end{array}$ & $\begin{array}{c}0(0) \\
2(12.5)\end{array}$ & 0.242 \\
\hline C-IMT right (mm) & $\begin{array}{l}1 \\
2\end{array}$ & $\begin{array}{l}16 \\
16\end{array}$ & $\begin{array}{l}0.65 \pm 0.15 \\
0.66 \pm 0.15\end{array}$ & 0.892 \\
\hline C-IMT left (mm) & $\begin{array}{l}1 \\
2\end{array}$ & $\begin{array}{l}16 \\
16\end{array}$ & $\begin{array}{l}0.65 \pm 0.16 \\
0.64 \pm 0.16\end{array}$ & 0.974 \\
\hline C-IMT maximum (mm) & $\begin{array}{l}1 \\
2\end{array}$ & $\begin{array}{l}16 \\
16 \\
\end{array}$ & $\begin{array}{l}0.65 \pm 0.16 \\
0.70 \pm 0.15\end{array}$ & 0.354 \\
\hline FMD & $\begin{array}{l}1 \\
2\end{array}$ & $\begin{array}{l}16 \\
16\end{array}$ & $\begin{array}{c}8.32 \pm 2.150 \\
8.25 \pm 2.99\end{array}$ & 0.934 \\
\hline
\end{tabular}

1, Simple snorers (Group 1); 2, mild OSAS (Group 2); T2DM, type 2 diabetes mellitus; C-IMT, carotid intima-media thickness; FMD, flow-mediated dilatation. 
and were reported as mean \pm standard deviation for continuous variables and as a percentage (\%) for categorical variables. The population was divided into two subgroups on the basis of the absence of OSAS (snorer patients, control group, Group 1) or the presence of mild OSAS (Group 2). Continuous variables presented Gaussian distribution and were compared with Student's $t$-test for independent samples and dichotomic variables with the Chi-squared test of Fisher. It was a twotailed test. We performed also an analysis of univariate binomial logistic regression, by considering as dependent variable the presence /absence of OSAS and as independent variables, respectively, all the parameters under study. The objective of the binomial logistic regression was to verify which study parameters were able to increase the odds ratio (OR) of OSA. The algorithm "K-Nearest Neighbors" (KNN) was applied to the measured parameters to investigate a possible grouping of samples. $\mathrm{KNN}$ is a non-parametric technique based on the determination of the distances between an unknown object and each of the objects in the training set. The $\mathrm{K}$ objects closer to the unknown sample are selected and a rule of majority was applied: the unknown is classified in the group in which a majority of the $\mathrm{K}$ objects belong. The method of classification was undertaken after applying the algorithm SELECT as a technique of selection function. We built Receiver Operating Characteristic (ROC) curves starting from the probability derived to a discriminant analysis to belong to the Group 2. For all statistical analyzes was assumed a statistically significant difference for $p<0.050$. All statistical analyzes were conducted with the software IBM SPSS Statistics 22.0.

\section{Results}

We enrolled 32 consecutive patients with a mean age $39.47 \pm 11.46$ years including 16 males and 16 females. The population was divided into 2 groups: Group 1 consisted of simple snorer patients with an average age of $39.375 \pm 12.054$ years and Group 2 consisted of patients with mild OSAS with an average age of $39.6 \pm 11.2$ years. Demographic and clinical characteristics of patients with mild OSAS and simple snorers are showed in Table 1. Both groups comprised non-obese subjects, and patients in Group 2 had a BMI basically higher than in group 1 , although not statistically significant $(25.9 \pm 4.8$ vs $22.8 \pm 3.2$; $\mathrm{p}=0.067)$ (Table 1$)$. Patients with mild OSAS also had serum total cholesterol values statistically significantly higher (178.625 \pm 24.878 vs $159.212 \pm 25.319$; $\mathrm{p}=0.038$ ) (Table 1$)$. By the contrast, no difference was found for other parameters between the two groups (Table 1). From the binomial logistic regression univariate analysis emerged as serum total cholesterol was a significant predictor of the probability of suffering of mild OSAS [OR: 1.03; $95 \%$ confidence interval (CI): 1.00-1.07; $\mathrm{p}=0.034$ ). The maximum C-IMT, although it did not reach the limits of statistical significance $(\mathrm{p}=0.344)$, presented an OR of 9.60 to belong to the Group 2 (Table 2). From the discriminant analysis, using as predictors the variable resulted significant within the binomial logistic regression (serum total cholesterol) and the main variable object of interest in the present study (maximum C-IMT), we obtained a good classification of the sample, with the system that correctly classified $68.8 \%$ of mild OSAS patients (Figure 1). The ROC curves, starting from the probability derived to the discriminant analysis to belong to the Group 2 , showed diagnostic accuracy that was statistically significant [area under the curve (AUC): 0.74, 95\% CI: 0.57-0.92, $\mathrm{p}=0.019$ ] (Figure 2).

Table 2. Analysis of binomial logistic regression univariate.

\begin{tabular}{lccc} 
& Odd ratio & IC 95\% & Significance \\
Cholesterol & 1.032 & $1.000-1.066$ & 0.048 \\
\hline IMT MAX & 9.603 & $0.89-1037.92$ & 0.344 \\
\hline
\end{tabular}

\section{Discussion}

In the present study, blood cholesterol values and the main parameter of our study, the cIMT, allow to clearly discriminate patients with OSA mild by non-OSA roncopathic patients. In the discriminant analysis, using as predictors, serum total cholesterol and the main variable object of interest in our study (maximum C-IMT), we obtained a good classification of the sample, with the system that correctly classified $68.8 \%$ of mild OSAS patients.

OSAS has been demonstrated to represent an important cardiovascular risk factor that would act by inducing oxidative stress and hypoxia as a major cause of endothelial dysfunction. OSAS also causes hyperactivity of the sympathetic nervous system and insulin resistance [24]. The assessment of C-IMT and FMD may provide important tools to predict cardiovascular risk, since the C-IMT is an expression of early alterations in the morphology of the vessel $[26,27]$ OSAS may cause endothelial dysfunction and abnormalities of FMD values [28]. In the present study, consisted of a group of patients at early stages of OSAS, we have verified how the cIMT and blood cholesterol values are able to correctly distinguish the minor osas from the snoring in $68 \%$ of the cases. The system also correctly classified almost $70 \%$ of mild OSAS patients demonstrating also excellent reliability and a significant diagnostic accuracy through ROC curves.

At present, OSAS is considered an independent factor of cardiovascular risk, as well as hypertension, diabetes, obesity and dyslipidemia. Prospective and cross-sectional studies suggested the OSAS may be a causal factor in the development of cardiovascular disease in obese patients with BMI $>30$ [3]. The mechanisms underlying the association between OSAS and cardiovascular disease are not yet completely understood, but many

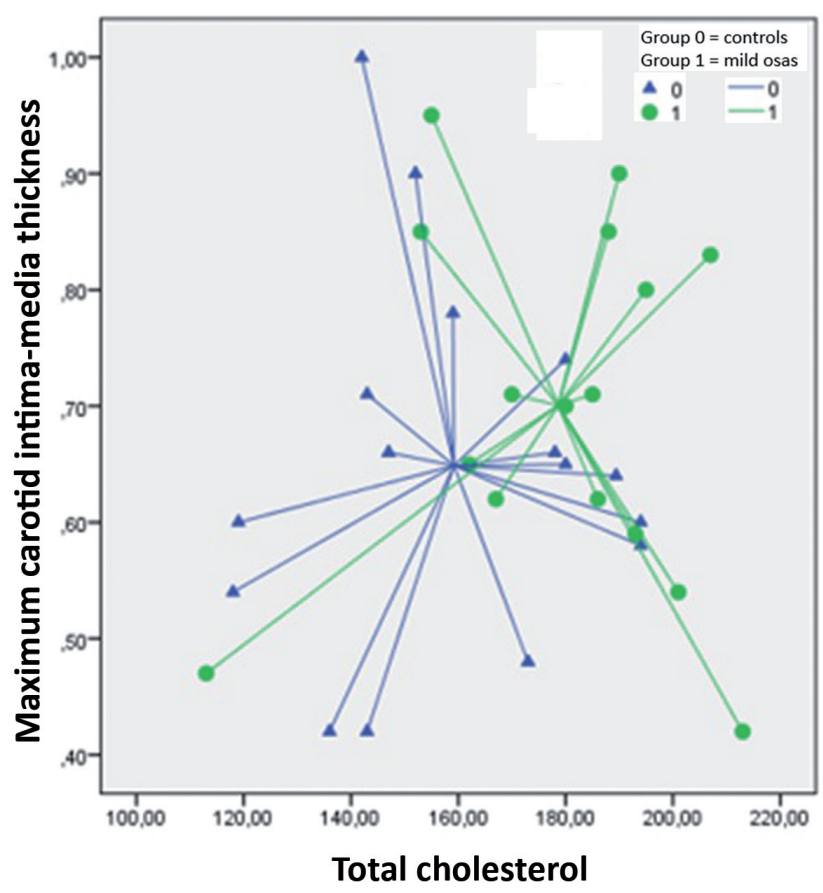

Figure 1. Discriminant analysis between the two groups of simple snorers (Group 1) and the patients with mild obstructive sleep apnea syndrome (OSAS) (Group 2) using as predictors total cholesterol and maximum carotid intima-media thickness (C-IMT). The system correctly classified $68.8 \%$ of cases. 
causal factors have been proposed that lead to the damage of the vessel wall and the formation of atherosclerotic plaques, i.e., reflex sympathetic activation, increases of blood pressure, hypercoagulability, microarousals, endothelial dysfunction, systemic inflammation and the action of reactive oxygen species [4]. All these mechanisms lead to inactivation of the nitric oxide (NO) by endothelial increased production of vasoconstrictor factors and free radicals of oxygen in the arterial wall $[10,29]$. In particular, endothelial dysfunction and increased rigidity of the arterial wall play a central role in the pathogenesis of atherosclerosis and are associated with cardiovascular risk factors [30,31]. Consequently, there is a growing interest for the measurement of these mechanisms associated with cardiovascular risk as early intervention would be beneficial to those patients who are at increased risk of future cardiovascular events. Numerous studies of obese patients associated moderate-severe OSAS with endothelial dysfunction, increased arterial stiffness and hypertension; conditions which, however, can improve after therapy with CPAP $[6,32,33]$. The clinical relevance of OSAS acquires increasing importance in relation to its chronic course, the long-term care that it requires, the increased risk for cardio-cerebrovascular diseases (4 times higher) and in terms of accidents at work by excessive daytime sleepiness.

Several studies have shown that even patients with minimally symptomatic OSAS have endothelial alteration and increased arterial stiffness, compared with control subjects without OSAS [34]. Altough this was not associated with a significant increase in blood pressure, the results suggested that obese patients with minimally symptomatic OSAS were at increased cardiovascular risk, as it was demonstrated in the most severe disease. Other studies have confirmed this hypothesis [35,36], in fact, it has been shown that in obese patients with mild OSAS and minimally symptomatic OSAS peripheral endothelial dysfunction was present. Furthermore, endothelial dysfunction was significantly reduced by the treatment with CPAP therapy [36]. In fact, moderate-severe OSAS with daytime symptoms, associated with endothelial dysfunction and increased arterial stiffness, improved after CPAP therapy [37-38]. However, we have no data from randomized controlled trials (RCTs) on the effects of CPAP on endothelial dysfunction and arterial stiffness in patients with mild OSAS. These minimally symptomatic patients usually do

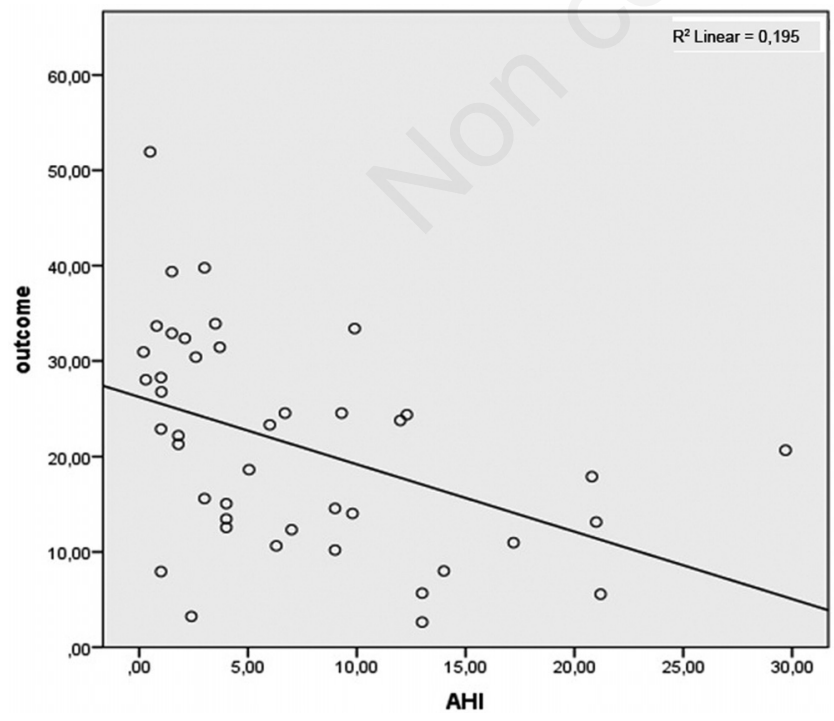

Figure 2. Receiver Operating Characteristic (ROC) curves starting from the probability derived from the discriminant analysis to belong to the group of patients with mild obstructive sleep apnea syndrome (OSAS) (Group 2). not receive CPAP therapy, as there is conflicting evidence of any significant benefit [39-41] or a reduction in blood pressure [42]. One study investigated the hypothesis that CPAP therapy may improve vascular function in obese patients with minimally symptomatic OSAS [14] evaluating the effects after 6 months of CPAP therapy on endothelial function and arterial stiffness in a subset of patients enrolled in the RCT Multicentre Obstructive Sleep Apnoea Interventional Cardiovascular (MOSAIC) [41]. This substudy of the MOSAIC trial showed that endothelial function improved after 6 months of CPAP therapy in patients with minimally symptomatic OSAS. The study showed that patients with minimally symptomatic OSAS can benefit of CPAP therapy at least in terms of improved function endothelial, although this treatment was not associated with a significant beneficial effect on arterial stiffness, blood pressure and cardiovascular risk score [36].

Recent studies have pointed out that endothelial function was not always preserved in obese patients with mild OSAS. However, its dysfunction may depend on the duration of the disease or may be increased by the coexistence of other important comorbidities [43]. This hypothesis was then confirmed by other studies showing that OSAS is a major cause of cardiovascular morbidity. Therefore, an intervention on lifestyle with weight reduction should be suggested to all obese patients with OSAS, not only as a first-line treatment for mild OSAS, but also included in the treatment protocol in patients with OSAS receiving CPAP. Furthermore, in most of the patients with mild OSAS, this was not diagnosed, so a better recognition of OSAS is vital, as with early intervention with weight reduction it is more likely that organs may still have the ability to completely revert metabolic and cardiovascular adverse effects or at least prevent the progression of the disease [44].

About the effects of CPAP therapy on various cardiac diseases associated with OSAS, an important study showed a decrease in mean arterial pressure of $9.9 \mathrm{mmHg}$ after 65 days of treatment with CPAP in hypertensive patients with OSAS [45]. CPAP treatment also may reduce the heart rate, probably by increasing the vagal tone and reducing the activation of the sympathetic nerve terminations [46]. The main clinical benefit of this reduction in heart rate is represented by a ratio intake /consumption of oxygen advantageous to the myocardium during application of CPAP. Finally, treatment with CPAP- therapy reduces atherosclerosis, not by changing the levels of serum lipids, but reducing inflammatory markers of vascular disease and sympathetic activation [9].

This study has some limitations. The first limitation is the reduced size of the sample. Furthermore, the size of the sample was not defined beforehand. We enrolled for 1 year all patients who met the inclusion and exclusion criteria of the study. Secondly, it is not a longitudinal study but a case-control study

\section{Conclusions}

This work highlights, to our knowledge for the first time, that blood cholesterol and cIMT values allow us to discriminate snoring patients from mild and no obese patients. In the early stages of OSAS, an alteration of lipid metabolism would appear to occur. These data are to be confirmed in longitudinal studies with a larger sample

\section{References}

1. Shimokawa H. Primary endothelial dysfunction: atherosclerosis. J Mol Cell Cardiol 1999;31:23-7.

2. Young T, Peppard PE, Gottlieb DJ. Epidemiology of obstructive sleep apnea: a population health perspective. Am J Respir Crit Care Med 2002;165:1217-39. 
3. Peker Y, Hedner J, Norum J, et al. Increased incidence cardiovascular disease in middle-aged men with obstructive sleep apnea: a 7-year follow-up. Am J Respir Crit Care Med 2002;166:159-65.

4. Somers VK, Kyken ME, Clary MP, Abbound FM. Sympathetic neural mechanisms in obstructive sleep apnea. J Clin Invest 1995;96:1897-904.

5. Shamsuzzaman AS, Winnicki M, Lanfranchi P, et al. Elevated C-reactive protein in patients with obstructive sleep apnea. Circulation 2002;105:2462-4.

6. Zhang CC, Wang ZG, Wu JM, et al. The laparoscopic nissen fundoplication eliminates obstructive sleep apnea syndrome due to gastroesophageal reflux disease. Ind J Surg 2013;75(Suppl 1):326-8.

7. Rezaeitalab F, Moharrari F, Saberi S, et al. The correlation of anxiety and depression with obstructive sleep apnea syndrome. J Res Med Sci 2014;19:205-10.

8. Epstein LJ, Kristo D, Strollo PJ Jr, et al. Adult Obstructive Sleep Apnea Task Force of the American Academy of Sleep Medicine. Clinical guideline for the evaluation, management and long-term care of obstructive sleep apnea in adults. J Clin Sleep Med 2009 $15 ; 5: 263-76$.

9. Drager LF, Bortolotto LA, Figueiredo AC, et al. Effects of continuous positive airway pressure on early signs of atherosclerosis in obstructive sleep apnea. Am J Respir Crit Care Med 2007;176:706-12.

10. Parish JM, Somers VK. Obstructive sleep apnea and cardiovascular disease. Mayo Clin Proc 2004;79:1036e46.

11. Ryan S, Taylor CT, McNicholas WT. Systemic inflammation: a key factor in the pathogenesis of cardiovascular complications in obstructive sleep apnoea syndrome? Thorax 2009;64:631e6.

12. Olson LJ, Olson EJ, Somers VK. Obstructive sleep apnea and platelet activation: another potential link between sleep disordered breathing and cardiovascular disease. Chest 2004;126:339e41.

13. Suzuki T, Nakano H, Maekawa J, et al. Obstructive sleep apnea and carotid-artery intima-media thickness. Sleep 2004;27:129e33.

14. Ip MS, Lam B, Ng MM, et al. Obstructive sleep apnea is independently associated with insulin resistance. Am J Respir Crit Care Med 2002; 165:670e6.

15. Laaban JP, Mounier L, Roque d'Orbcastel 0, et al. Cardiovascular risk factors in men and women with obstructive sleep apnea syndrome. Respir Med 2010;104:1063e8.

16. American Academy of Sleep Medicine. International classification of sleep disorders. In: Diagnostic and Coding Manual. 2nd ed. Westchester, IL: American Academy of Sleep Medicine; 2005.

17. European Association for Cardiovascular Prevention \& Rehabilitation, Reiner Z, Catapano AL, et al. ESC/EAS Guidelines for the management of dyslipidaemias: the Task Force for the management of dyslipidaemias of the European Society of Cardiology (ESC) and the European Atherosclerosis Society (EAS). Eur Heart J 2011;32:1769-818.

18. Mancia G, Fagard R, Narkiewicz K, et al. ESH/ESC guidelines for the management of arterial hypertension: the Task Force for the Management of Arterial Hypertension of the European Society of Hypertension (ESH) and of the European Society of Cardiology (ESC). Eur Heart J 2013;34:2159-219.

19. Authors/Task Force Members, Rydén L, Grant PJ, et al. ESC Guidelines on diabetes, pre-diabetes, and cardiovascular diseases developed in collaboration with the EASD: the Task Force on diabetes, pre-diabetes, and cardiovascular diseases of the European Society of Cardiology (ESC) and developed in collaboration with the European Association for the Study of Diabetes (EASD). Eur Heart J 2013;34:3035-87.

20. Friedewald WT, Levy RI, Fredrickson DS. Estimation of the concentration of low-density lipoprotein cholesterol in plasma, without use of the preparative ultracentrifuge. Clin Chem. 1972;18:499 502.
21. Berry RB, Budhiraja R, Gottlieb DJ, et al. Rules for scoring respiratory events in sleep: Update of the 2007 AASM Manual for the scoring of sleep and associated events. J Clin Sleep Med 2012;8:597-619.

22. Sateia MJ. International classification of sleep disorders-third edition: highlights and modifications. Chest 2014;146:1387-94.

23. Gemignani V, Faita F, Ghiadoni L, et al. A system for real-time measurement of the brachial artery diameter in B-mode ultrasound images. IEEE Trans Med Imaging 2007;26:393-404.

24. Pignoli P, Tremoli E, Poli A, et al. Intimal plus medial thickness of the arterial wall: a direct measurement with ultrasound imaging. Circulation 1986;74:1399-406.

25. Corretti MC, Anderson TJ, Benjamin EJ, et al. Guidelines for the ultrasound assessment of endothelial-dependent flowmediated vasodilation of the brachial artery: a report of the International Brachial Artery Reactivity Task Force. J Am Coll Cardiol 2002;39:257-65.

26. Lorenz MW, Markus HS, Bots ML, et al. Prediction of clinical cardiovascular events with carotid intima-media thickness. A systematic review and meta-analysis. Circulation 2007;115:459e67.

27. Ciccone MM, Balbarini A, Porcelli MT, et al. Carotid artery intimamedia thickness: normal and percentile values in the Italian population (CAMP study). Eur J Cardiovasc Prev Rehabil 2011;18:650e5.

28. Araújo Lda S, Fernandes JF, Klein MR, Sanjuliani AF. Obstructive sleep apnea is independently associated with inflammation and insulin resistance, but not with blood pressure, plasma catecholamines, and endothelial function in obese subjects. Nutrition 2015;31:1351-7.

29. Carratù $\mathrm{P}$, Ventura VA, Maniscalco $\mathrm{M}$, et al. Echocardiographic findings and plasma endothelin-1 levels in obese patients with and without obstructive sleep apnea. Sleep Breath 2016;20:613-9.

30. Celermajer DS, Sorensen KE, Gooch VM, et al. Non-invasive detection of endothelial dysfunction in children and adults at risk of atherosclerosis. Lancet 1992;340:1111-5.

31. Schachinger V, Britten MB, Zeiher AM. Prognostic impact of coronary vasodilator dysfunction on adverse long-term outcome of coronary heart disease. Circulation 2000;101:1899-906.

32. Pepperell JCT, Ramdassingh-Dow S, Crosthwaite N, et al. Ambulatory blood pressure after therapeutic and subtherapeutic nasal continuous positive airway pressure for obstructive sleep apnoea: a randomised parallel trial. Lancet 2002;359:204-10.

33. Kohler M, Pepperell JCT, Casadei B, et al. Effects of continuous positive airway pressure on arterial stiffness, sympathetic activity, and baroreflex sensitivity in obstructive sleep apnea: a randomized controlled trial [abstract]. Am J Respir Crit Care Med 2008;177:A292.

34. Kohler M, Craig S, Nicoll D, et al. Endothelial function and arterial stiffness in minimally symptomatic obstructive sleep apnea. Am J Respir Crit Care Med 2008;178:984-8.

35. Ciccone MM, Favale S, Scicchitano P, et al. Reversibility of the endothelial dysfunction after CPAP therapy in OSAS patients. Int $\mathrm{J}$ Cardiol 2012;158:383-6.

36. Kohler M, Craig S, Pepperell JC, et al. CPAP improves endothelial function in patients with minimally symptomatic OSA: results from a subset study of the MOSAIC trial. Chest 2013;144:896-902.

37. Kohler M, Pepperell JCT, Casadei B, et al. CPAP and measures of cardiovascular risk in males with OSAS. Eur Respir J 2008;32:1488-96.

38. Robinson GV, Smith DM, Langford BA, et al. Continuous positive airway pressure does not reduce blood pressure in nonsleepy hypertensive OSA patients. Eur Respir J 2006;27:1229-35.

39. Craig SE, Kohler M, Nicoll D, et al. Continuous positive air- way pressure improves sleepiness but not calculated vascular risk in patients with minimally symptomatic obstructive sleep apnoea: the MOSAIC randomised controlled trial. Thorax 2012;67:1090-6. 
40. Monasterio C, Vidal S, Duran J, et al. Effectiveness of continuous positive airway pressure in mild sleep apnea-hypopnea syndrome. Am J Respir Crit Care Med 2001;164:939-43.

41. Barbé F, Mayoralas LR, Duran J, et al. Treatment with continuous positive airway pressure is not effective in patients with sleep apnea but no daytime sleepiness: a randomized, controlled trial. Ann Intern Med 2001;134:1015-23.

42. Barbé F, Durán-Cantolla J, Capote F, et al. Spanish Sleep and Breathing Group. Long-term effect of continuous positive airway pressure in hypertensive patients with sleep apnea. Am J Respi Crit Care Med 2010;181:718-26.

43. Carratù $\mathrm{P}$, Ciccone MM, Dragonieri S, Resta 0 . Endothelial func- tion is not always well preserved in obese patients with mild OSA. Sleep Breath 2015;19:15.

44. Blomster H, Tuomilehto $\mathrm{H}$. Author response to: endothelial function is not always well preserved in obese patients with mild OSA. Sleep Breath 2015;19:17-8.

45. Becker HF, Jerrentrup A, Ploch T, et al. Effect of nasal continuous positive airway pressure treatment on blood pressure in patients with obstructive sleep apnea. Circulation 2003;107:68-73.

46. Naughton MT, Rahman MA, Hara K, et al. Effect of continuous positive airway pressure on intrathoracic and left ventricular transmural pressures in patients with congestive heart failure. Circulation 1995;91:1725-31. 